\title{
Minireview
}

\section{The effects of lipids on channel function}

\author{
Anthony G Lee
}

Address: School of Biological Sciences, University of Southampton, Southampton, SO16 7PX, UK. Email: agl@soton.ac.uk

\section{Abstract}

Anionic lipids affect the function of many channels, including connexins, as shown in a recent report in BMC Biology. These effects might follow from direct binding of the anionic lipids to the channels.

\section{Lipids affect channel function}

Many membrane channels, for proper function, require the presence of particular species of lipid in the surrounding membrane. In a recent paper in BMC Biology, Locke and Harris [1] provide an example of this: they showed that connexin channels are inactive in a membrane composed of just the zwitterionic lipid phosphatidylcholine (PC) but become active in the presence of the anionic lipid phosphatidylserine (PS) (for lipid structures see Figure 1). The full effect of PS is seen only when 60\% or more of the lipid is PS. The effect is not structurally specific; other anionic lipids such as phosphatidic acid (PA) and phosphatidylinositol (PI) have the same effect as PS. Similar observations have been made with other channels. For example, the probability that the potassium channel KcsA is open is very low in bilayers of PC alone but increases with increasing anionic lipid content [2]; the functions of inward rectifying potassium channels are modulated by PIs [3]; and the presence of anionic lipid increases the rate of flux of small molecules through the mechanosensitive channel MscL [4].

\section{Lipid binding to channels}

How are the effects of anionic lipid on channel function to be understood? In some cases the interaction of lipid with a membrane protein is highly specific. For example, a single molecule of the anionic lipid phosphatidylglycerol is seen in the X-ray crystal structure of the heterotrimeric nitrate reductase $\mathrm{A}$, bound in a distinct pocket formed by all three subunits, with three positively charged residues contributing to the binding site [5]. In this case binding is strong as well as structurally specific, because the lipid remains bound during the process of crystallization from detergent solution. However, most lipid molecules interacting with a membrane protein are not buried within the structure in this way, but are located on the transmembrane surface of the protein, forming a ring or annulus around the protein [6]. These annular lipid molecules 'solvate' the transmembrane surface of a membrane protein in the same way that water molecules solvate the surface of a water-soluble protein. Given that the effects of anionic lipid on channel function are generally seen only at high concentrations of the anionic lipid, it is likely that the

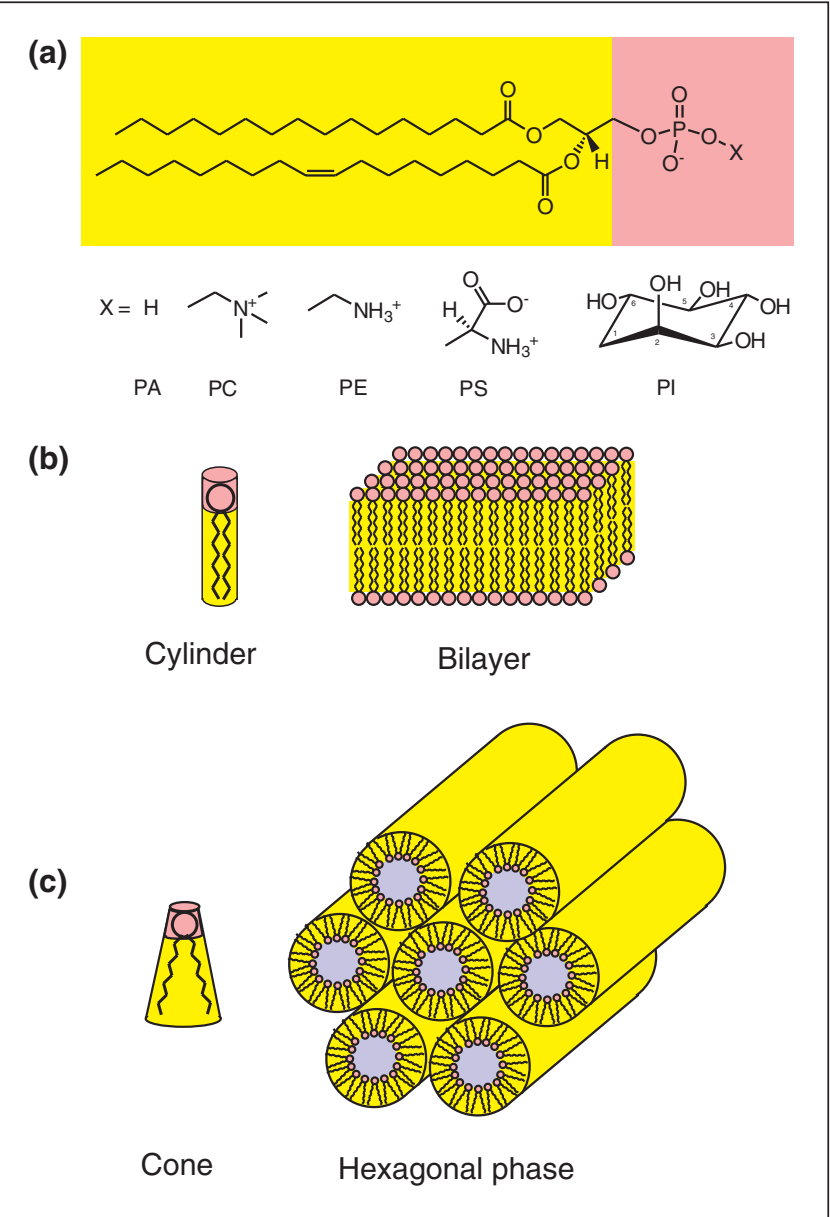

Figure 1

Lipid structures and phases. (a) The structure of the major glycerophospholipids phosphatidic acid (PA), phosphatidylcholine (PC), phosphatidylethanolamine (PE), phosphatidylserine (PS) and phosphatidylinositol $(\mathrm{PI})$, which differ only in the group indicated by $X$. Yellow indicates the hydrophobic tail; orange indicates the charged headgroup, which can be anionic or, where there is a positive and a negative charge, zwitterionic. (b) Lipids with a cylindrical shape, such as PC, pack as bilayers. (c) Lipids with a conical shape, such as PA or PE, pack in curved structures such as the hexagonal $\mathrm{H}_{\|}$phase. 
(a)

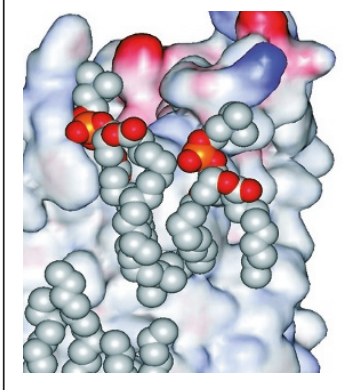

(b)

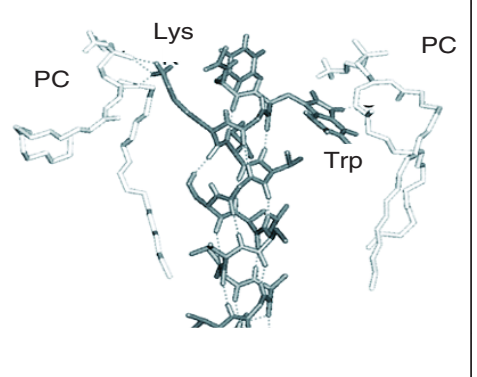

Figure 2

Annular lipids. (a) Part of the transmembrane surface of aquaporin showing bound annular lipid molecules. The protein is shown as a surface plot colored by charge (blue, positive; red, negative), with the lipid molecules shown in space-filling format (red, oxygen; orange, phosphorus; grey, carbon). From Protein DataBank (PDB) code 2B6O. (b) A snapshot from a molecular dynamics simulation of a transmembrane $\alpha$-helix in a lipid bilayer. The helix is the sixth transmembrane $\alpha$-helix of the potassium channel Kv. The lysine side chain can be seen hydrogen bonding to phosphate and ester oxygens of a phosphatidylcholine (PC) molecule, and the tryptophan side chain forms a hydrogen bond to an ester oxygen. Modified from [8].

relevant lipid-protein interactions are relatively weak, and that the observed effects are caused by anionic lipids binding at annular sites on the channel.

Although annular lipid molecules are seldom seen in X-ray crystal structures, they are seen in a few special cases, such as in the crystalline arrays formed by aquaporins (Figure 2a) [7]. The annular lipid molecules are seen to be highly distorted to match the rough surface of the protein. Interactions of the lipid headgroups with the protein involve a set of weak charge and hydrogen bonded interactions, as shown in the molecular dynamics simulation in Figure 2b [8].

Binding of lipid molecules at annular sites is described by a set of exchange reactions at each site. For example, an annular site on a membrane protein located in a bilayer containing a mixture of PC and PS could be occupied by either a PC or by a PS molecule:

$$
\text { Protein-PC + PS } \leftrightarrows \text { Protein-PS + PC }
$$

This exchange reaction is defined by an equilibrium constant $K$, the relative lipid binding constant for PS compared with PC:

$$
K=[\text { Protein-PS }][\mathrm{PC}] /[\text { Protein-PC][PS }]
$$

Slightly stronger binding of PS than PC at a particular site $(K>1)$ will ensure that the site is occupied mostly by PS at the relatively high concentrations of anionic lipid required to affect channel function. For example, in a bilayer containing $60 \%$ PS, an annular site will be $90 \%$ occupied by PS if binding of PS at the site is just five times stronger than binding of PC. This binding would affect channel function if it resulted in a significant conformational change in the channel.

A functionally important annular lipid binding with a preference for anionic lipids has been demonstrated on MscL, for which the 'hot spot' for binding anionic lipid was shown to correspond to a cluster of three positively charged residues [4]; it is likely that similar clusters of positively charged residues close to the lipid-water interface occur on other membrane proteins. In their recent paper, Locke and Harris [1] report a mass spectrometric method for detecting specificity in lipid binding to connexin channels. They expressed connexin26 and connexin32 in HeLa cells, isolated hemichannels (that is, channels that had not yet formed gap junctions linking apposed cells) using a nonionic detergent, and characterized the lipids remaining with the hemichannel preparation; lipids associated with a gap junction preparation were also analyzed. Although all classes of lipid were found associated with the hemichannels, there was an enrichment in anionic lipids, consistent with interaction with clusters of positively charged residues of the type suggested above. Indeed, the crystal structure of connexin26 shows a concentration of positively charged residues at the lipid-water interface, on the intracellular side [9].

Locke and Harris [1] also found that lipids with particular combinations of fatty acyl chains remained with the purified connexin26 samples, whereas lipids with other combinations of fatty acyl chains remained with the purified connexin32 samples. It would be surprising if this reflected chain-specific binding to the connexins because the effects of chain structure on lipid binding to membrane proteins are generally small [6]. Another possibility, as described by Locke and Harris [1], is that the fatty acyl chain compositions of the cells expressing connexin26 and connexion32 are slightly different.

\section{Collective physical properties}

Because high concentrations of anionic lipid are needed to affect channel function, there is another possible mechanism for the effects of anionic lipid that needs to be considered. Large changes in the chemical composition of a bilayer will result in changes in collective physical properties of the bilayer, such as its thickness and fluidity, and in the related properties of spontaneous curvature and pressure profile across the membrane [6]; it is therefore conceivable that any observed changes in channel function follow from changes in these collective physical properties rather than from changes in the pattern of charge and hydrogen-bonding interactions between the lipids and the proteins described above. 


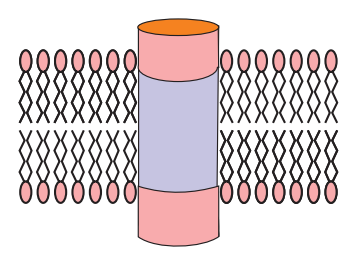

Closed

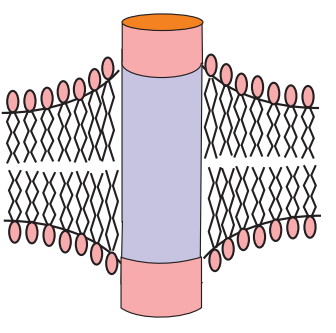

Open
Figure 3

How spontaneous curvature of lipids could affect channel function. The hydrophobic thickness of the lipid bilayer matches that of the channel in the closed state, but the hydrophobic thickness of the channel could increase on channel opening. This would require stretching of the lipid bilayer around the protein to maintain hydrophobic matching, and the associated curving of the bilayer would be easier if the lipid bilayer contains lipids that prefer to adopt a curved structure.

Recently, interest has focused on the spontaneous curvature of the membrane. Spontaneous curvature relates to the idea that lipids have a distinct 'shape' (Figure 1). A lipid such as PC, in which the lipid headgroup and the two fatty acyl chains occupy roughly equal areas in the plane of the lipid bilayer, has a cylindrical shape, and so packs well into a bilayer (Figure 1b). However, a lipid such as PA or phosphatidylethanolamine (PE), for which the area of the headgroup is small relative to that of the two chains, has a conical shape and will prefer to pack into a curved structure such as the hexagonal $\mathrm{H}_{\mathrm{II}}$ phase (Figure 1c); these lipids have a spontaneous curvature, whereas PC does not. One way in which it has been proposed that this could have a significant effect on protein function is illustrated in Figure 3. If the thickness of the transmembrane region of a channel were to increase on channel opening, the bilayer would have to stretch around the open channel to prevent exposure of hydrophobic groups to water. This stretching, because it involves a curving of the membrane in the vicinity of the protein, would be favored by a lipid such as PA or PE that favors a curved structure. The explanation requires, of course, that there should be a significant change in hydrophobic thickness for the channel on opening and there is, as yet, no evidence that this is the case. Nevertheless, membrane protein function has often been observed to be different in PE and in PC, a difference that could be attributed to the different spontaneous curvatures of PE and PC. However, studies of the effects of lipids with headgroups intermediate between those of PE and PC, with one and two methyl groups, suggest that what is important is not spontaneous curvature but the ability to hydrogen bond $[4,10]$.

What conclusions can be drawn from these studies? It is clear that the presence of anionic lipids, generally at high concentrations, affects the function of a wide range of channels. In MscL the anionic lipids bind to a cluster of positively charged residues close to the lipid-water interface, and similar charge interactions are likely to be important for other channels. It is also likely that the effects of anionic lipids on channel function follow directly from binding to these charge clusters, although the possible importance of the collective physical properties of the lipid bilayer cannot yet be ruled out.

\section{References}

1. Locke D, Harris AL: Connexin channels and phospholipids: association and modulation. BMC Biol 2009, 7:52.

2. Marius $P$, Zagnoni M, Sandison ME, East JM, Morgan H, Lee AG: Binding of anionic lipids to at least three nonannular sites on the potassium channel KcsA is required for channel opening. Biophys $J$ 2008, 94:1689-1698.

3. Fan Z, Makielski JC: Anionic phospholipids activate ATPsensitive potassium channels. J Biol Chem 1997, 272:53885395.

4. Powl AM, East JM, Lee AG: Importance of direct interactions with lipids for the function of the mechanosensitive channel MscL. Biochemistry 2008, 47:12175-12184.

5. Bertero MG, Rothery RA, Palak M, Hou C, Lim D, Blasco F, Weiner JH, Strynadka NCJ: Insights into the respiratory electron transfer pathway from the structure of nitrate reductase A. Nat Struct Biol 2003, 10:681-687.

6. Lee AG: How lipids affect the activities of integral membrane proteins. Biochim Biophys Acta 2004, 1666:62-87.

7. Gonen T, Cheng YF, Sliz P, Hiroaki Y, Fujiyoshi Y, Harrison SC, Walz T: Lipid-protein interactions in double-layered twodimensional AQPO crystals. Nature 2005, 438:633-638.

8. Shrivastava IH, Capener CE, Forrest LR, Sansom MSP: Structure and dynamics of $\mathrm{K}^{+}$channel pore-lining helices: a comparative simulation study. Biophys J 2000, 78:79-92.

9. Maeda S, Nakagawa S, Suga M, Yamashita E, Oshima A, Fujiyoshi Y, Tsukihara T: Structure of the connexin 26 gap function channel at 3.5 $\AA$ resolution. Nature 2009, 458:597602.

10. Hakizimana P, Masureel M, Gbaguidi B, Ruysschaert JM, Govaerts C: Interactions between phosphatidylethanolamine headgroup and LmrP, a multidrug transporter. J Biol Chem 2008, 283:9369-9376.

Published: 6 October 2009

doi:10.1186/jbiol178

(c) 2009 BioMed Central Ltd 